\title{
Perancangan Belt Konveyor Kapasitas 25 TPH (Studi Kasus : PT. Bumi Mineral Sulawesi)
}

\author{
Muas M. ${ }^{*}$, Syaharuddin R. ${ }^{2}$, Arwin S. ${ }^{3}$ dan Alfian N. ${ }^{4}$ \\ 1,2,3,4 Jurusan Teknik Mesin, Politeknik Negeri Ujung Pandang, Makassar 90245, Indonesia \\ *muas@poliupg.ac.id
}

\begin{abstract}
This study aims to (1) To determine the workload of the conveyor belt system (2) To determine the distance between idlers (3) To determine the slope of the conveyor belt (4) To determine the driving motor power. The design of the 25 TPH conveyor belt is a problem at PT. Bumi Mineral Sulawesi. That is because, one of the components in the conveyor belt equipment does not yet exist. The intended component is the motor drive on all belt conveyor passes (planned to have 4 belt conveyor passes). The results of this activity have been obtained on belt conveyor 1 ( $B C$ 01) the material to be destroyed is rock crushed with a density of $2 \mathrm{~T} / \mathrm{m} 3$, belt length of 17 meters, conveyor belt capacity of $25 \mathrm{TPH}$, belt slope of 170, belt width of $500 \mathrm{~mm}$, the maximum belt voltage is $7,806 \mathrm{kN} / \mathrm{m}$, the distance between the carrying idler is $1200 \mathrm{~mm}$, the distance between the return idler is 3000 $\mathrm{mm}$, with a motor power of $5.5 \mathrm{~kW}$. In conveyor belt 2 (BC 02) the material to be destroyed is rock crushed with a density of $2 \mathrm{~T} / \mathrm{m} 3$, belt length of 13 meters, conveyor belt capacity of $25 \mathrm{TPH}$, belt slope of 170 , belt width of 500 $\mathrm{mm}$, belt maximum voltage of 7,806 kN/m distance between carrying idlers $1200 \mathrm{~mm}$, distance between return idlers $3000 \mathrm{~mm}$, with motor power of $5.5 \mathrm{~kW}$. On conveyor belts 03 and 04 (BC 03 and BC 04) the material to be destroyed is rock crushed with a density of $2 \mathrm{~T} / \mathrm{m} 3$, belt length of 10 meters, conveyor belt capacity of $25 \mathrm{TPH}$, belt slope of 170, belt width of $500 \mathrm{~mm}$, maximum belt voltage 7,806 kN/m distance between carrying idlers 1200 $\mathrm{mm}$, distance between return idlers $3000 \mathrm{~mm}$, with $5.5 \mathrm{~kW}$ motor power
\end{abstract}

Keywords: Stone Crusher; Belt Conveyor; Driving Motor; Idler

\begin{abstract}
Abstrak: Penelitian ini bertujuan untuk (1) Untuk mengetahui beban kerja dari sistem belt konveyor (2) Untuk mengetahui jarak antar idler (3) Untuk mengetahui kemiringan belt conveyor (4) Untuk mengetahui daya motor penggerak. Perancangan belt conveyor kapasitas $25 \mathrm{TPH}$ ini menjadi permasalahan di $\mathrm{PT}^{1}$. Bumi Mineral Sulawesi. Hal tersebut disebabkan, salah satu komponen pada peralatan belt conveyor belum ada. Komponen yang dimaksud yakni motor penggerak pada semua lintasan belt conveyor (direncanakan ada 4 lintasan belt conveyor). Hasil dari kegiatan ini telah didapatkan pada belt conveyor 1 (BC 01) material yang akan dihancurakan adalah batu (rock crushed) dengan massa jenis $2 \mathrm{~T} / \mathrm{m} 3$, panjang belt 17 meter, kapasitas belt conveyor $25 \mathrm{TPH}$, Kemiringan belt 170, lebar belt $500 \mathrm{~mm}$, tegangan maksimum belt 7,806 kN/m jarak antar carrying idler $1200 \mathrm{~mm}$, jarak antar return idler $3000 \mathrm{~mm}$, dengan daya motor 5,5 kW. Pada belt conveyor 2 (BC 02) material yang akan dihancurakan adalah batu (rock crushed) dengan massa jenis $2 \mathrm{~T} / \mathrm{m} 3$, panjang belt 13 meter, kapasitas belt conveyor $25 \mathrm{TPH}$, Kemiringan belt 170, lebar belt $500 \mathrm{~mm}$, tegangan maksimum belt 7,806 $\mathrm{kN} / \mathrm{m}$ jarak antar carrying idler $1200 \mathrm{~mm}$, jarak antar return idler $3000 \mathrm{~mm}$, dengan daya motor 5,5 kW. Pada belt conveyor 03 dan 04 (BC 03 dan BC 04) material yang akan dihancurakan adalah batu (rock crushed) dengan massa jenis $2 \mathrm{~T} / \mathrm{m} 3$, panjang belt 10 meter, kapasitas belt conveyor $25 \mathrm{TPH}$, Kemiringan belt 170, lebar belt 500 $\mathrm{mm}$, tegangan maksimum belt 7,806 kN/m jarak antar carrying idler $1200 \mathrm{~mm}$, jarak antar return idler $3000 \mathrm{~mm}$, dengan daya motor $5,5 \mathrm{~kW}$.
\end{abstract}

Kata kunci : Stone Crusher; Belt Conveyor; Motor Penggerak; Idler

\section{PENDAhuluan}

Belt conveyor merupakan salah satu jenis pemindah bahan yang beroperasi secara kontinu dan mengikuti jalur atau lintasan tetap. Belt conveyor dapat mengangkut material secara mendatar atau dengan kemiringan tertentu, baik material yang berupa unit load maupun bulk material [1]. Unit load adalah material yang dapat dihitung jumlahnya satu per satu, misalnya: kotak, kantong, balok, dsb. 
Bulk material adalah material yang berbentuk butir-butir, bubuk atau serbuk, misalnya: batuan kecil, semen, pasir, dsb [2].

PT. Bumi Mineral Sulawesi (PT. BMS) melakukan sebuah langkah taktis bagi perusahaan dengan membeli sebuah alat modern sistem penghancur batu atau sering disebut stone crusher system. Stone crusher sistem terdiri atas sejumlah peralatan, yakni: feeder dan hopper, crusher (jaw crusher), belt conveyor, scalping unit, grizzly bar. Alat ini sebelumnya telah dioperasikan oleh perusahaan asing asal Tiongkok. Pembelian tersebut selain untuk mempermudah pekerjaan bagi perusahaan pada umumnya dan karyawan pada khususnya, juga merupakan sebuah langkah untuk memperkecil pengeluaran perusahaan ataupun merupakan sebuah keuntungan tersendiri pada perusahaan.

Permasalahan yang muncul dalam pembelian ini, PT. BMS belum bisa mengoperasikan ataupun merakit stone crusher system tersebut. Hal tersebut disebabkan, salah satu komponen pada peralatan belt conveyor belum ada. Komponen yang dimaksud yakni motor penggerak pada semua lintasan belt conveyor (direncanakan ada 4 lintasan belt conveyor). Oleh karena itu, agar alat dapat beroperasi dengan efisien dan produktif, perusahaan harus merencanakan terlebih dahulu kapasitas daya motor per lintasan yang dibutuhkan untuk menggerakkan belt conveyor.

Selain itu, agar alat tersebut bisa bekerja secara optimal, perusahaan juga harus merencanakan beberapa masalah vital lainnya, yakni: material yang akan digunakan, kapasitas daya angkat belt conveyor, jarak idler pada stone crusher, sudut kemiringan pada belt conveyor, tegangan belt conveyor, diameter puli serta, kapasitas daya motor pada belt conveyor, untuk menopang kapasitas 25 TPH yang akan dicapai.

Mekanisme perhitungan kapasitas daya motor beserta parameter lainnya, yang akan digunakan umumnya mengacu pada conveyor equipment manufacturers association [3] dan berikut adalah masalah yang menurut kami sangat perlu untuk diteiliti agar optimasi kinerja alat ini stabil yaitu, bagaimana menganalisis beban kerja dari sistem belt konveyor, bagaimana menganalisis jarak antar idler, bagaimana menganalisis kemiringan belt conveyor, bagaimana menganalisis daya motor penggerak. Agar pembahasan masalah mengenai penelitian ini tidak terlalu melebar, maka kami penulis memberi batasan permasalahan yaitu, Material yang akan diangkut oleh belt conveyor, jarak antar idler, kapasitas belt conveyor yang digunakan disesuaikan dengan kapasitas stone crusher, kemiringan belt conveyor, tegangan belt conveyor, diameter pully yang akan digunakan pada belt conveyor dan daya motor yang akan digunakan untuk penggerak belt conveyor. Adapun manfaat dari penelitian ini yaitu, diketahuinya material yang akan dihancurkan, diketahuinya jarak antar idler, diketahuinya kemiringan belt conveyor, diketahuinya diameter puli yang akan digunakan dan diketahuinya kapasitas daya motor untuk penggerak belt conveyor.

\section{METODE PENELITIAN}

Penelitian dilaksanakan di perusahaan PT. Bumi Mineral Sulawesi yang berlokasi di Jalan Poros Makassar - Palopo Km.356 Dusun Lamone Desa Karang-Karangan Kecamatan Bua, Luwu, Sulawesi Selatan.

Berikut diagram alir metode penelitian ini, yaitu dengan mendeskripsikan langkah-langkah penelitian dari awal sampai selesai. 
61 Muas M, Syaharuddin R, Arwin S, Alfian N. Perancangan Belt Konveyor Kapasitas 25 TPH (Studi Kasus : PT. Bumi Mineral Sulawesi)

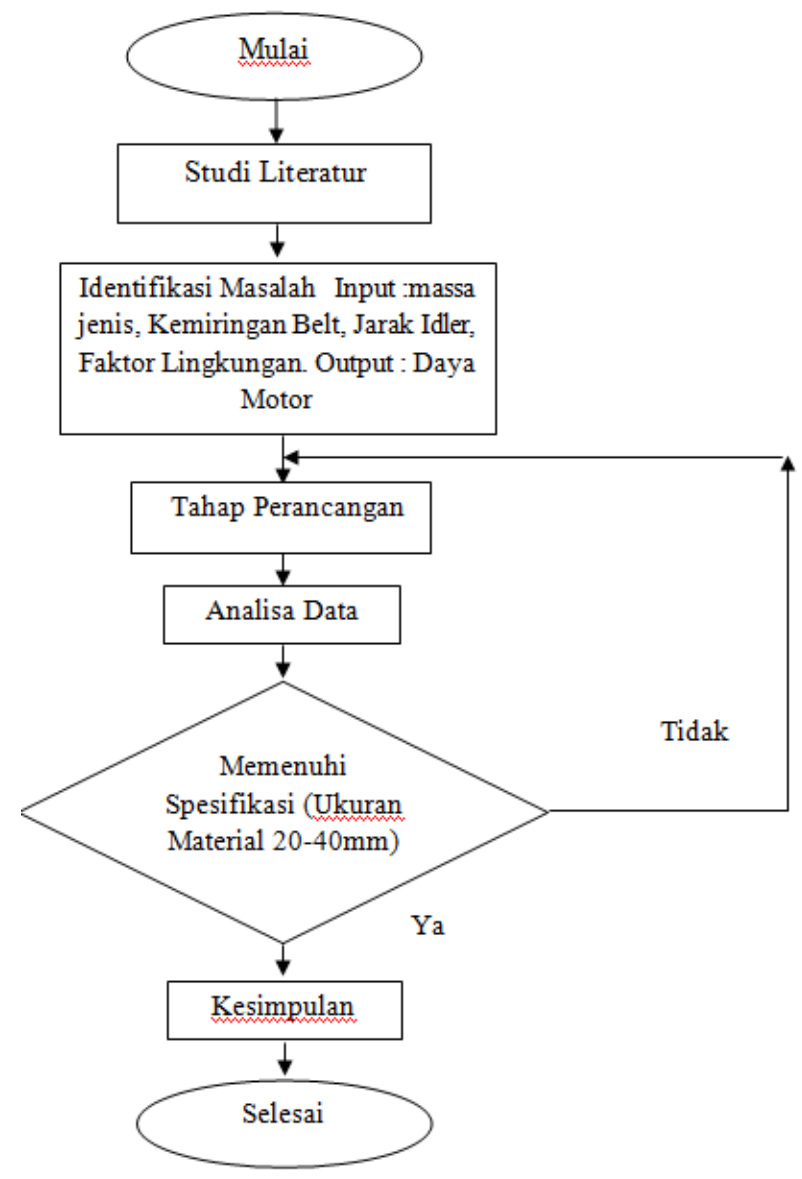

Gambar 1. Flow Chart Metode Penelitian

\section{HASIL DAN PEMBAHASAN}

Desain (Gambar 2) ini ditentukan atas berbagai pertimbangan sebagai berikut :

1. BC 01 Tipe BW $500 \times 17$ m untuk menghantar material dari Jaw Crusher [4] ke Vibrating.

2. BC 02 Tipe BW $500 \times 13 \mathrm{~m}$ untuk menghantar material yang ukurannya lebih besar dari $40 \mathrm{~mm}$ kembali ke Jaw Crusher.

3. BC 03 \& 04 Tipe BW $500 \times 10$ untuk menghantar material yang berukuran $20-40 \mathrm{~mm} \mathrm{ke}$ stockpile.

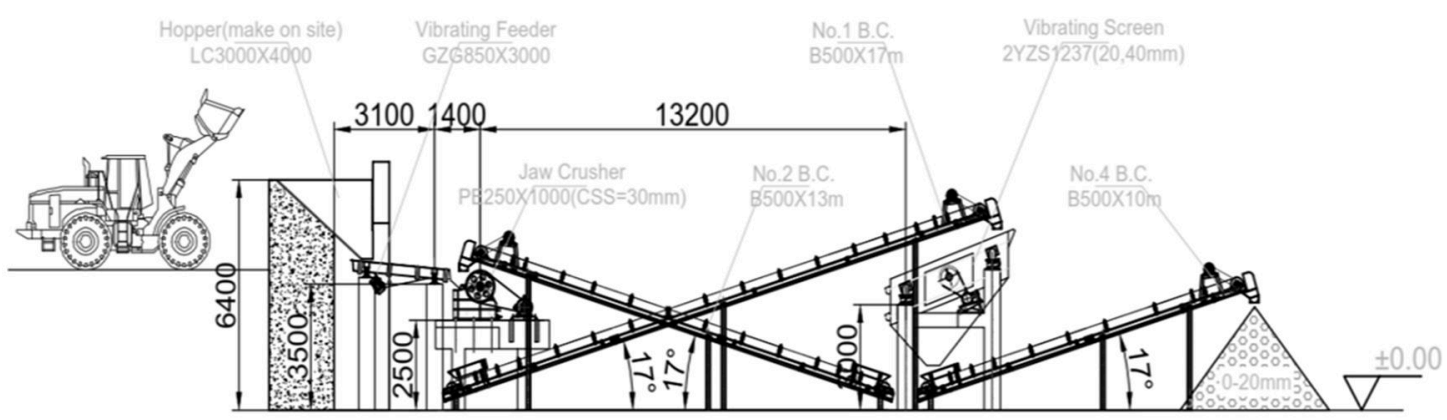

Gambar 2. Design Konstruksi Stone Crusher Kapasitas 25 TPH 
Untuk menetukan kapasitas motor Belt Conveyor dengan kapasitas 25 TPH diperlukan beberapa parameter sebagai berikut :

- Material yang diangkut (rock crushed)

- $\quad$ Massa jenis material $(\mathrm{d})=2 \mathrm{~T} / \mathrm{m} 3$

- Surcharge angle $(\mathrm{sa})=25 \mathrm{deg}$

- $\quad$ Troughing angle $(a)=35 \mathrm{deg}$

- Kapasitas belt conveyor $(\mathrm{Q})=25 \mathrm{TPH}$

- $\operatorname{Design}$ capacity $(\mathrm{T})=\mathrm{Q} \times 1,1=25 \mathrm{TPH} \times 1,1=27,5$

- Kecepatan belt. $(\mathrm{V})=1,5 \mathrm{~m} / \mathrm{s}$

- Area beban cross section $(A)=0,040877338 \mathrm{~m} 2$

- Lebar belt $(\mathrm{b})=500 \mathrm{~mm}=0,5 \mathrm{~m}$

- Carrying Idler $(\mathrm{Si})=1200 \mathrm{~mm}=1,2 \mathrm{~m}$

- Return Idler $(\mathrm{Sr})=3000 \mathrm{~mm}=3 \mathrm{~m}$

- Massa belt $(W b)=7,5 \mathrm{Kg} / \mathrm{m}$

A. Perhitungan Belt Conveyor 01 (BC-01) [5]

Direncanakan : $\mathrm{L}=17 \mathrm{~m}$

a. Massa material batu persatuan panjang (Wm) $W m=0,278 \times T V=0,278 \times 27,51,5 \mathrm{~m} / \mathrm{s}=5,10 \mathrm{Kg} / \mathrm{m}$

b. Total beban idler persatuan panjang (Wi)

c. Idler Selection

$$
W i=W m+W b \quad=5,10 \mathrm{Kgm}+75 \mathrm{Kgm} \quad=12,60 \mathrm{Kg} / \mathrm{m}
$$

Service factor $(\mathrm{K} 3)=1,1$

Diameter roll belt yang dipilih $=4$ inch

Factor koreksi kecepatan belt $(\mathrm{K} 4)=0,8$

Beban actual idler (IL)

$$
I L=(W b+W m) S i=(5,10 \mathrm{kgm}+7,5 \mathrm{kgm}) 1,2 \mathrm{~m}=(12,60 \mathrm{kgm}) 1,2 \mathrm{~m}=15,12 \mathrm{~kg}
$$

Beban yang disesuaikan (AL)

$$
A L=I L \times K 1 \times K 2 \times K 3 \times K 4=15,12 \times 0,1 \times 1,1 \times 1,1 \times 0,8=14,6
$$

Dengan menggunakan nilai terbesar antara idler load (IL) dan adjusted load (AL) untuk idler yang layak dari beban idler dengan melihat Tabel CEMA 5-8 sampai 5-12 sehingga diperoleh 15,12 [6].

Factor gesekan carrying idler $(\mathrm{Kx})$

$$
\begin{aligned}
& K x=1,7 \times(0.00068(W b+W m)+A i / S i)=1,7 \times(0.00068(7,5+5,10)+0,821,2) \\
& K x=1,7 \times(0,00068(1,60)+0,68) \\
& K x=1,171 \mathrm{Kg}^{2} / \mathrm{m}^{2}
\end{aligned}
$$

Faktor gesekan skirtboard $(\mathrm{Cs})=0,256$

Conveyor c/c horizontal length $(\mathrm{L})=17$ meter

Conveyor c/c along belt $(\mathrm{Lx})=L \operatorname{Cos} K L x=17 \operatorname{Cos} 17 \mathrm{Lx}=17,777$ meter

Tinggi conveyor (H1)

$$
M=\tan 17(17 \pi / 180) M=0,3505(17 \pi 180)=5,197 \text { meter }
$$

Total ketinggian conveyor $(\mathrm{H} 1+\mathrm{H} 2)=5,197+0=5,197$ meter

d. Perhitungan tegangan belt conveyor

Jumlah discharge plow $=0$

Jumlah dari loading points $(\mathrm{Ni})=1$

Panjang Skritboard $(\mathrm{Lb})=3$ meter

Jumlah dari pembersih belt $(\mathrm{Nbc} 1)=0$

Jumlah dari $V$ belt cleaner $(\mathrm{Nbc} 2)=1$

Jumlah dari Type Pulley A (NA) $=1$ Head Pully

Jumlah dari Type Pulley B (NB) $=1$ Tail and Take up pulley 
63 Muas M, Syaharuddin R, Arwin S, Alfian N. Perancangan Belt Konveyor Kapasitas 25 TPH (Studi Kasus : PT. Bumi Mineral Sulawesi)

Jumlah dari Type Pulley $\mathrm{C}(\mathrm{NC})=0$ Bend and snap pulley

Faktor gesekan belt conveyor dan material di atas idler $(\mathrm{Ky})=0,031$

Tegangan minimal di starting point carry run

(T3) or (Tt or To)

$T 3=6,25 \times(\mathrm{Wb}+\mathrm{Wm}) \times$ Si $\times 9,807=6,25 \times(7,5+5,10) \times 1,2 \times 9,80$

$T 3=6,25 \times 12,60 \times 1,2 \times 9,807$

$T 3=932,468 \mathrm{~N}$

Tegangan akibat dari gesekan dan point carry run (Tfcx) $T f c x=L x x(K x+K y$

$(W b+W m)) \times 9,807 T$

$f c x=17,777 \times(1,171+0,031(7,5+5,10)) \times 9,807$

$T f c x=222,366 \mathrm{~N}$

Tegangan karena beban pada titik akhir carry run (Twcx) Twcx $=H x x$

$(W b+W m) \times 9,807$

$T w c x=5,197 \times(7,5+5,10) \times 9,807=642,066 \mathrm{~N}$

Tegangan karena gesekan pada titik akhir carry run (Tcx)

$T c x=T 3+T f c x+T w c x=932,468+222,366+642,066=1796,901 N$

Tegangan karena resistansi pulley (Tp)

$T p=(N A \times 90+N B \times 68+N C \times 45) \times 9,807$

$T p=(1 \times 90+1 \times 68+0 \times 45) \times 9,807$

$T p=1549,506 \mathrm{~N}$

Tegangan karena akselerasi material (Tam)

Tam $=0,278 \times$ Ni $\times$ T $\times$ V Tam $=0,278 \times 1 \times 27,5 \times 1,5$

Tam $=11,468 \mathrm{~N}$

Tegangan karena skirtboard friction (Tsb)

$T s b=14,6 \times L b \times\left(10,765 \times C s \times h s^{2}+6\right)$

$T s b=14,6 \times 3 \times(10,765 \times 0,276 \times 0,0802+6)$

$T s b=263,633 N$

Tegangan karena double bladed belt cleaner (Tbc1)

$T b c 1=N b c 1 \times 875 \times b$

$T b c 1=0 \times 875 \times 0,5$

$T b c 1=0 N$

Tegangan karena type $V$ belt cleaner $(\mathrm{Tbc} 2)$

$T b c 2=N b c 2 \times 525 \times b=1 \times 525 \times 0.5=262,500 \mathrm{~N}$

Tegangan karena Discharge Plow (Tpl)

$T p l=(N p l \times B p \times b) \times 9,807=(0 \times 89,290 \times 0,5) \times 9,807=0 N 39$

Total tegangan pada titik akhir carry run (T11)

$T 11=T c x+T p+T a m+T s b+T b c 1+T b c 2$

$T 11=1796,901+1549,506+11,468+263,633+0+262,500=3884,007 N$

Tegangan karena belt sag - Tsg

$T s g=6,25 \times(W b+W m) \times$ Si $\times 9,807=6,25 \times(7,5+5,10) \times 1,2 \times 9,807$ $=1773,792 \mathrm{~N}$

Tegangan karena berat belt dan return belt $-\mathrm{Tb}-\mathrm{N}$

$T b=H x \times W b \times 9,807=5,197 \times 7,5 \times 9,807=354,217 N$

Tegangan karena gesekan dari return idlers $-T y x-N$

Tyx $=\operatorname{Lx} \times 0,015 \times$ Wb $x$ Kt $\times 9,807=17,777 \times 0,015 \times 7,5 \times 1,0 \times 9,807$

Tyx $=19,613 \mathrm{~N}$

Slack side tension due to belt sag etc. $N$

$T 21=T s g+T b-T y x=1773,792+354,217-19,613=2108,396 N$ 
Slack side tension due to belt wrap (T22)

$$
T 22=C w \times T E=1,20 \times 1774,171=2129,005 \mathrm{~N}
$$

Tight side tension (T1)

$$
T 1=T E+T 2=1774,171+2129,005=3903,175 \mathrm{~N}
$$

Tegangan maximum belt conveyot (Tmax)

$$
\operatorname{Tmax}=(0.001 \times T 1) / b=(0,001 \times 3903,175) 0,5=7,806 \mathrm{kN} / \mathrm{m}
$$

e. Pemilihan belt

Karena tegangan maksimum belt yang terjadi lebih kecil dari pada tegangan izin belt, maka tegangan belt aman terhadap tegangan yang diizinkan.

Tmax $<$ T izin

$7.806 \mathrm{kN} / \mathrm{m}<50 \mathrm{kN} / \mathrm{m}$

f. Pemilihan Motor [7]

Daya yang digunakan untuk penggerak belt $(\mathrm{PB})$

$$
P B=0,001 \times T E \times V=0,001 \times 1774,171 \times 1,5=2,661 \mathrm{~kW}
$$

Drive Pulley loss

$$
P L=1 \times 90 \times 9,807 \times 0,001 \times V=1 \times 90 \times 9,807 \times 0,001 \times 1,5=1,324 \mathrm{~kW}
$$

Kecepatan reduction loss $(P R)$

$$
P R=5 \%(\mathrm{~PB}+\mathrm{PL})=5 \%(2,661+1,324)=0.199 \mathrm{~kW}
$$

Daya yang dibutuhkan pada poros motor (Pshaft)

$$
\text { Pshaft }=\mathrm{PB}+\mathrm{PL}+\mathrm{PR}=2,661+1,324+0,199=4,184 \mathrm{~kW}
$$

Daya minimum motor yang akan digunakan

$$
P m=\text { Pshaft } \times E f=4,184 \times 0,9=4,649 \mathrm{~kW}
$$

Berdasarkan hasil perhitungan daya motor yang akan digunakan untuk mengerakkan belt conveyor 1 (BC 01) sepanjang 17 meter dengan kemiringan belt conveyor 17o adalah 4,649 $\mathrm{kW}$. Dan berdasarkan dengan daya motor yang ada di pasaran tidak terdapat daya 4,649 maka kami memilih motor dengan daya $5,5 \mathrm{~kW}$.

\section{B. Perhitungan Belt Conveyor 2 (BC-02)}

Direncanakan : $\mathrm{L}=13 \mathrm{~m}$

a. Massa material Batu (Wm)

$W m=0,278 \times T V=0,278 \times 27,51,5 \mathrm{~m} / \mathrm{s}=5,10 \mathrm{Kg} / \mathrm{m}$

b. Total beban idler (Wi)

$W i=W m+W b=5,10 \mathrm{Kgm}+75 \mathrm{Kgm} \quad=12,60 \mathrm{Kg} / \mathrm{m}$

c. Idler Selection

Lump adjustment factor $(K 1)=0,1$

Service factor $(\mathrm{K} 3)=1.1$

Kecepatan belt yang dipilih berdasarkan kecepatan design $(\mathrm{v})=1,136 \mathrm{~m} / \mathrm{s}$.

Diameter roll belt yang dipilih $=4$ inch

Factor koreksi kecepatan belt $(\mathrm{K} 4)=0,8$

Beban actual idler (IL)

$\mathrm{IL}=(\mathrm{Wb}+\mathrm{Wm}) \mathrm{Si}=(5,10 \mathrm{kgm}+7,5 \mathrm{kgm}) 1,2 \mathrm{~m}=(12,60 \mathrm{kgm}) 1,2 \mathrm{~m}=15,12 \mathrm{~kg}$

Beban yang disesuaikan (AL)

$$
A L=I L \times K 1 \times K 2 \times K 3 \times K 4=15,12 \times 0,1 \times 1,1 \times 1,1 \times 0,8=14,6
$$


65 Muas M, Syaharuddin R, Arwin S, Alfian N. Perancangan Belt Konveyor Kapasitas 25 TPH (Studi Kasus : PT. Bumi Mineral Sulawesi)

Dengan menggunakan nilai terbesar antara idler load (IL) dan adjusted load (AL) untuk idler yang layak dari beban idler dengan melihat Tabel CEMA 5-8 sampai 5-12 sehingga diperoleh 15,12 [6].

Diameter idler rolls selected $=5$ inch

Force to rotated idler $($ Ai $)=0,82 \mathrm{~kg}$

Factor gesekan carrying idler $(\mathrm{Kx})$

$$
\begin{aligned}
& \mathrm{Kx}=1,7 \times(0,00068(W b+W m)+A i / S i) \\
& \mathrm{Kx}=1,7 \times(0,00068(7,5+5,10)+0.821 .2)=1,7 \times(0,00068(12,60)+0,68) \\
& \mathrm{Kx}=1,171 \mathrm{Kg} 2 / \mathrm{m} 2
\end{aligned}
$$

Faktor gesekan Skirtboard $(\mathrm{Cs})=0,256$

Kemiringan belt conveyor $(\mathrm{K})=17$

Conveyor c/c along belt (Lx)

$$
L x=L \operatorname{Cos} K=13 \operatorname{Cos} 17=13,594 \text { meter }
$$

Tinggi belt conveyor $(\mathrm{H} 1)$

$$
M=\tan 13(17 \pi / 180)=0,231(17 \pi 180)=3,974 \text { meter }
$$

Tinggi total belt conveyor $(\mathrm{H} 1+\mathrm{H} 2)=3,974+0=3,974$ meter

d. Perhitungan tegangan belt

Jumlah dari loading points $(\mathrm{Ni})=1$

Panjang Skritboard $(\mathrm{Lb})=3$ meter

Jumlah dari double bladed belt cleaner $(\mathrm{Nbc} 1)=0$

Jumlah dari $V$ belt cleaner $(\mathrm{Nbc} 2)=1$

Jumlah dari Type Pulley A (NA) = 1 Head Pully

Jumlah dari Type Pulley B (NB) $=1$ Tail and Take up pulley

Jumlah dari Type Pulley $\mathrm{C}(\mathrm{NC})=0$ Bend and snap pulley

Factor gesekan dari belt dan material di atas idler $(\mathrm{Ky})=0,031$

Tegangan minimal di titik awal carry run (T3) or (Tt or To)

$$
\begin{aligned}
& T 3=6,25 \times(W b+W m) \times \text { Si } \times 9,807=6,25 \times(7,5+5,10) \times 1,2 \times 9,807 \\
& T 3=6,25 \times 12,60 \times 1,2 \times 9,807=932,468 N
\end{aligned}
$$

Tegangan karena gesekan di titik carry run (Tfcx)

$$
\begin{aligned}
& T f c x=L x x(K x+K y \quad(W b+W m)) 9,807 \\
& T f C x=13,594 x(1,171+0,031(7,5+5,10)) x 9,807=168,998 N
\end{aligned}
$$

Teganngan karena beban di akhir carry run (Twcx)

$$
\begin{aligned}
& T w c x=H x \times(W b+W m) 9,807 \\
& T w c x=3,974 x(7,5+5,10) \times 9,807=490,992 N
\end{aligned}
$$

Tegangan karena gesekan di akhir carry run (Tcx)

$$
T c x=T 3+T f c x+T w c x=932,468+168,998+490,992=1592,458 N
$$

Tegangan karena resistansi dari pully $(\mathrm{Tp})$

$$
\begin{aligned}
& T p=(N A \times 90+N B \times 68+N C \times 45) \times 9,807 \\
& T p=(1 \times 90+1 \times 68+0 \times 45) \times 9,807=1549,506 N
\end{aligned}
$$

Tegangan karena akselerasi material. (Tam)

$$
\text { Tam }=0,278 \times N i \times T \times V=0,278 \times 1 \times 27,5 \times 1,5 \text { Tam }=11,468 \mathrm{~N}
$$

Tegangan karena gesekan skirtboard (Tsb)

$$
\begin{aligned}
& T s b=14,6 \times L b \times\left(10,765 \times C s \times h s^{2}+6\right) \\
& T s b=14,6 \times 3 \times\left(10,765 \times 0,276 \times 0,080^{2}+6\right)=263,633 N
\end{aligned}
$$


Tegangan karena double bladed belt cleaner (Tbc1)

$T b c 1=N b c 1 \times 875 \times b=0 \times 875 \times 0,5=0 N$

Tegangan karena type $\mathrm{V}$ belt cleaner $($ Tbc2)

$T b c 2=N b c 2 \times 525 \times b=1 \times 525 \times 0,5=262,500 N$

Tegangan karena Discharge Plow (Tpl)

$T p l=(N p l \times B p \times b) \times 9,807=(0 \times 89,290 \times 0,5) \times 9,807=0 N$

Total tegangan di titik akhir Carry run (T11)

$$
\begin{aligned}
& T 11=T c x+T p+T a m+T s b+T b c 1+T b c 2 \\
& T 11=1592,458+1549,506+11,468+263,633+0+262,500 \\
& T 11=3679,565 N
\end{aligned}
$$

Tegangan karena belt sag - (Tsg)

Tsg $=6,25 \times(W b+W m) \times$ Si $x 9,807=6,25 \times(7,5+5,10) \times 1,2 \times 9,807$

Ts $g=1773,792 \mathrm{~N}$

Tegangan karena $\mathrm{Wb}$. of return belt (Tb)

$T b=H \times X W b 9 ., 07=3,974 \times 7,5 \times 9,807=270,872 \mathrm{~N}$

Tegangan karena gesekan return idlers (Tyx)

Tyx $=\operatorname{Lx} \times 0,015 \times$ Wb $x$ Kt $\times 9,807=13,594 \times 0,015 \times 7,5 \times 1,0 \times 9,807$

Tyx $=13,897 \mathrm{~N}$

Slack side tension due to belt sag etc.

$$
\begin{aligned}
& T 21=T s g+T b-T y x=1773,792+270,872-13,897=2030,767 \\
& T E=T 11-T 21=3697,565-2030,767=1648,797
\end{aligned}
$$

Slack side tension due to belt wrap (T22)

$T 22=C w \times T E=1,20 \times 1648,797=1978,556 N$

Tight side tension $(\mathrm{T} 1)$

$$
T 1=T E+T 2=1648,797+1978,556=3679,564 N
$$

Tegangan maksimum belt (Tmax)

$T \max =(0,001 \times T 1) / b=(0,001 \times 3679,564) 0,5=7,359 \mathrm{kN} / \mathrm{m}$

e. Pemilihan belt

Karena tegangan maksimum belt yang terjadi lebih kecil dari pada tegangan izin belt, maka tegangan belt aman terhadap tegangan belt yang diizinkan.

Tmax $<\mathrm{T}$ izin

$7.806 \mathrm{kN} / \mathrm{m}<50 \mathrm{kN} / \mathrm{m}$

f. Pemilihan Motor

Daya yang digunakan untk penggerak belt $(\mathrm{PB})=k W$

$$
P B=0,001 \times T E \times V=0,001 \times 1648,797 \times 1,5=2,473 \mathrm{~kW}
$$

Drive Pulley loss $(\mathrm{PL})=k W$

$$
P L=1 \times 90 \times 9,807 \times 0.001 \times V=1 \times 90 \times 9,807 \times 0,001 \times 1,5=1,324 \mathrm{~kW}
$$

Kecepatan reducer $(P R)=k W$

$$
\mathrm{PR}=5 \%(\mathrm{~PB}+\mathrm{PL})=5 \%(2.473+1.324)=0,190 \mathrm{~kW}
$$

Daya yang dibutuhkan pada poros motor (Pshaft) $=k W$

$$
\text { Pshaft }=\text { PB }+ \text { PL }+ \text { PR }=2,473+1,324+0,190=3,987 \mathrm{~kW}
$$

Daya minimum motor yang akan digunakan

$$
\text { Pm=Pshaft } \times E f=3,987 \times 0,9=4,440 \mathrm{~kW}
$$


67 Muas M, Syaharuddin R, Arwin S, Alfian N. Perancangan Belt Konveyor Kapasitas 25 TPH (Studi Kasus : PT. Bumi Mineral Sulawesi)

Berdasarkan hasil perhitungan daya motor yang akan digunakan untuk mengerakkan belt conveyor 1 (BC 01) sepanjang 13 meter dengan kemiringan belt conveyor 17o adalah 4,440 kW. Dan berdasarkan dengan daya motor yang ada di pasaran tidak terdapat daya 4,440 maka kami memilih motor dengan daya $5,5 \mathrm{~kW}$.

C. Perhitungan Belt Conveyor 03 (BC-03) [5]

Direncanakan : $\mathrm{L}=10 \mathrm{~m}$

a. Massa material $(\mathrm{Wm})$

$W m=0,278 \times T V=0,278 \times 27,51,5 \mathrm{~m} / \mathrm{s}=5,10 \mathrm{Kg} / \mathrm{m}$

b. Total beban idler (Wi)

$W i=W m+W b \quad=5,10 \mathrm{Kgm}+75 \mathrm{Kgm}=12,60 \mathrm{Kg} / \mathrm{m}$

c. Idler Selection

Lump adjustment factor $(K 1)=0,1$

Service factor $(\mathrm{K} 3)=1,1$

Faktor koreksi kecepatan belt $(\mathrm{K} 4)=0,8$

Beban actual idler (IL)

$I L=(W b+W m) S i=(5,10 \mathrm{kgm}+7,5 \mathrm{kgm}) 1.2 \mathrm{~m}=(12,60 \mathrm{kgm}) 1,2 \mathrm{~m}=15,12 \mathrm{~kg}$

Beban yang disesuaikan (AL)

$A L=I L \times K 1 \times K 2 \times K 3 \times K 4=15,12 \times 0,1 \times 1,1 \times 1,1 \times 0,8=14,6$

Dengan menggunakan nilai terbesar antara idler load (IL) dan adjusted load (AL) untuk idler yang layak dari beban idler dengan melihat Tabel CEMA 5-8 sampai 5-12 sehingga diperoleh 15,12 [6].

Faktor gesekan Carrying idler $(\mathrm{Kx})$

$$
\begin{aligned}
& K x=1,7 \times(0,00068(\mathrm{~Wb}+\mathrm{Wm})+\mathrm{Ai} / \mathrm{Si}) \\
& K x=1,7 \times(0,00068(7,5+5,10)+0,821,2)=1,7 \times(0,00068(12,60)+0,68) \\
& K x=1,171 \mathrm{Kg} 2 / \mathrm{m} 2
\end{aligned}
$$

Faktor gesekan Skirtboard $(\mathrm{Cs})=0,256$

Panjang Conveyor c/c horizontal $(\mathrm{L})=10$ meter

Conveyor c/c along belt (Lx) $L x=L \operatorname{Cos} K$

$$
L x=10 \operatorname{Cos} 17=10,457 \text { meter }
$$

Tinggi conveyor $(\mathrm{H} 1)$

$$
M=\tan 10(17 \pi / 180) M=0,176(17 \pi 180)=3,057 \text { meter }
$$

Tripper lifting $(\mathrm{H} 2)=0$ (tidak ada)

Total ketiggian belt conveyor

$$
(\mathrm{H} 1+\mathrm{H} 2)=3,057+0=3,057 \text { meter }
$$

d. Tension Calculation

Jumlah dari discharge plow $=0$

Jumlah dari loading points $(\mathrm{Ni})=1$

Panjang Skritboard $(\mathrm{Lb})=3$ meter

Jumlah dari double bladed belt cleaner $(\mathrm{Nbc} 1)=0$

Jumlah dari $V$ belt cleaner $(\mathrm{Nbc} 2)=1$

Jumlah dari Type Pulley A (NA) $=1$ Head Pully

Jumlah dari Type Pulley B (NB) $=1$ Tail and Take up pulley

Jumlah dari Type Pulley $\mathrm{C}(\mathrm{NC})=0$ Bend and snap pulley 
Faktor gesekan dari belt dan materialdi atas idler $(\mathrm{Ky})=0,031$

Tegangan minimal di titik awal carry run (T3) or (Tt or To)

$$
\begin{aligned}
& T 3=6,25 \times(W b+W m) \times \text { Si } \times 9,807 \\
& T 3=6,25 \times(7,5+5,10) \times 1,2 \times 9,807=6,5 \times 12,60 \times 1,2 \times 9,807 \\
& T 3=932,468 \mathrm{~N}
\end{aligned}
$$

Tegangan dari gesekan di titik carry run (Tfcx) $T f c x=L x x(K x+K y$

$$
(W b+W m)) 9.807 T f c x=10,457 x(1,171+0,031(7,5+5,10)) x
$$

9,807 Tf $c x=129,395 \mathrm{~N}$

Tegangan dari beban di titik akhir carry run $($ Twcx $)=$ Newton

$T w c x=H x \times(W b+W m) 9,807=3,057 \times(7,5+5,10) x 9,807=377,686 N$

Tegangan dari gesekan dan beban di titik akhir carry run $(\mathrm{Tcx})=\mathrm{N}$

$T c x=T 3+T f c x+T w c x=932,468+129,395+377,686=1439,549 \mathrm{~N}$

Tegangan darri resistansi pulley $(\mathrm{Tp})$

$T p=(N A \times 90+N B \times 68+N C \times 45) 9,807$

$T p=(1 \times 90+1 \times 68+0 \times 45) 9,807=1549,506 \mathrm{~N}$

Tegangan dari akselerasi material. (Tam)

Tam $=0,278 \times N i \times T \times V=0,278 \times 1 \times 27,5 \times 1,5$ Tam $=11,468 \mathrm{~N}$

Tegangan dari gesekan skirtboard (Tsb)

$T s b=14,6 \times \operatorname{Lb} \times\left(10,765 \times C s \times h s^{2}+6\right)$

$T s b=14,6 \times 3 \times(10,765 \times 0,276 \times 0,0802+6)=263,633 \mathrm{~N}$

Tegangan dari double bladed belt cleaner (Tbc1)

$T b c 1=N b c 1 \times 875 \times b=0 \times 875 \times 0,5=0 \mathrm{~N}$

Tegangan darit tipe $\mathrm{V}$ belt cleaner (Tbc2)

$T b c 2=N b c 2 \times 525 \times b=1 \times 525 \times 0,5=262,500 \mathrm{~N}$

Tegangan dari Discharge Plow $(\mathrm{Tpl})$

$T p l=(N p l \times B p \times b) \times 9,807=(0 \times 89,290 \times 0,5) \times 9,807=0 N$

Total tegangan di titik akhir Carry run (T11)

$T 11=T c x+T p+T a m+T s b+T b c 1+T b c 2$

$T 11=1439,549+1549,506+11,468+263,633+0+262,500$

$T 11=3526,655 \mathrm{~N}$

Tegangan dari belt sag (Tsg)

Ts $g=6,25 \times(W b+W m) \times$ Si $\times 9,807=625 \times(7,5+5,10) \times 1,2 \times 9,807$

$T s g=1773,792 \mathrm{~N}$

Tegangan dari $W b$. of return belt $(\mathrm{Tb})$

$T b=H \times \times W b \times 9,807=3,057 \times 7,5 \times 9,807=224,85 \mathrm{~N}$

Tegangan dari gesekan return idlers (Tyx)

Tyx $=$ Lx $x 0,015 \times$ Wb $x$ Kt $\times 9,807=10,457 \times 0,015 \times 7,5 \times 1,0 \times 9,807$

Tyx $=11,537 \mathrm{~N}$

Tegangan Slack side due to belt sag etc.

$W$

$$
T 21=T s g+T b-T y x=1773,792+224,85-11,537=1987,105
$$

$$
T E=T 11-T 21=3526,665-1987,105=1539,56
$$

Slack side tension due to belt wrap (T22)

$T 22=C w \times T E=1,20 \times 1539,56 T 22=1847,472 \mathrm{~N}$

Tight side tension (T1)

$$
T 1=T E+T 2=1539,56+1971,465=3511,025 \mathrm{~N}
$$

Tegangan maksimum belt (Tmax)

e. Pemilihan belt

$T \max =(0,001 \times T 1) / b=(0,001 \times 3511,025) 0,5=7,022 \mathrm{kN} / \mathrm{m}$ 
69 Muas M, Syaharuddin R, Arwin S, Alfian N. Perancangan Belt Konveyor Kapasitas 25 TPH (Studi Kasus : PT. Bumi Mineral Sulawesi)

Karena tegangan maksimum belt yang terjadi lebih kecil dari pada tegangan izin belt, maka tegangan belt aman terhadap tegangan belt yang diizinkan.

Tmax $<\mathrm{T}$ izin

$7.806 \mathrm{kN} / \mathrm{m}<50 \mathrm{kN} / \mathrm{m}$

f. Pemilihan motor [7]

Daya yang digunakan untuk penggerak belt $(\mathrm{PB})=k W$

$$
P B=0,001 \times T E \times V=0,001 \times 1539,56 \times 1,5=2,309 \mathrm{~kW}
$$

Drive Pulley loss (PL) $=k W$

$$
P L=1 \times 90 \times 9,807 \times 0,001 \times V=1 \times 90 \times 9,807 \times 0,001 \times 1,5=1,324 \mathrm{~kW}
$$

Kecepatan reducer $(P R)=k W$

$$
P R=5 \%(\mathrm{~PB}+\mathrm{PL})=5 \%(2,309+1,324)=0,181 \mathrm{~kW}
$$

Daya motor yang akan digunakan (Pshaft) $=\mathrm{kW}$

$$
\text { Pshaft }=\mathrm{PB}+\mathrm{PL}+\mathrm{PR}=2,309+1,324+0,181=3,814 \mathrm{~kW}
$$

Daya minimum motor yang akan digunakan

$$
P m=\text { Pshaftx } E f=3,8140,9=4,237 \mathrm{~kW}
$$

Berdasarkan hasil perhitungan daya motor yang akan digunakan untuk mengerakkan belt conveyor 1 (BC 01) sepanjang 17 meter dengan kemiringan belt conveyor 17o adalah 4.237 $\mathrm{kW}$. Dan berdasarkan dengan daya motor yang ada di pasaran tidak terdapat daya 4.237maka kami memilih motor dengan daya $5,5 \mathrm{~kW}$.

\section{Perhitungan Belt Conveyor 04 (BC 04)}

Sehubungan dengan ini type belt conveyor 03 dan belt conveyor 04 memiliki spesifikasi yang sama sehingga daya motor yang digunakan untuk menggerakan belt conveyor 04 adalah 5,5 $\mathbf{k W}$.

\section{KESIMPULAN}

Dari hasil analisa teori yang telah dilakukan, kami dapat menyimpulkan bahwa :

a. Hasil perhitungan daya motor untuk mengerakkan belt conveyor 1 (BC 01) sepanjang 17 meter dengan kemiringan belt conveyor 170 , jarak antar idler 1,2 meter dan tegangan maksimum yang terjadi pada belt sebesar $7.806 \mathrm{kN} / \mathrm{m}$ maka motor yang digunakan sebesar $\mathbf{5 , 5} \mathbf{~ k W}$.

b. Hasil perhitungan daya motor untuk mengerakkan belt conveyor 2 (BC 02) sepanjang 13 meter dengan kemiringan belt conveyor 170 , jarak antar idler 1,2 meter dan tegangan maksimum yang terjadi pada belt sebesar $7.806 \mathrm{kN} / \mathrm{m}$ maka motor yang digunakan sebesar $\mathbf{5 , 5} \mathbf{~ k W}$.

c. Hasil perhitungan daya motor untuk mengerakkan belt conveyor 3 (BC 03) dan 4 (BC 04 sepanjang 10 meter dengan kemiringan belt conveyor 170, jarak antar idler 1,2 meter dan tegangan maksimum yang terjadi pada belt sebesar $7.806 \mathrm{kN} / \mathrm{m}$ maka motor yang digunakan sebesar $\mathbf{5 , 5} \mathbf{~ k W}$.

\section{DAFTAR PUSTAKA}

[1] D. Poirier, "Conveyor dryers," in Handbook of Industrial Drying, Fourth Edition, 2014.

[2] T. F. George, R. R. Letfullin, and G. Zhang, Bulk metallic glasses. 2011.

[3] M. S. Lozier, "Deconstructing the conveyor belt," Science. 2010.

[4] A. Gupta and D. Yan, "Chapter 4 - Jaw Crusher," in Mineral Processing Design and Operations - 2nd Edition, 2016.

[5] G. Velmurugan, E. Palaniswamy, M. Sambathkumar, R. Vijayakumar, and T. M. Sakthimuruga, "Conveyor Belt Troubles (Bulk Material Handling)," Int. J. Emerg. Eng. Res. Technol., 2014.

[6] R. T. C. SWINDERMAN and C. E. M. Association, "Belt Conveyors for Bulk Materials," Chaners Publ. Company, Inc, vol. 6, p. 567, 2014.

[7] R. Nur and M. A. Suyuti, Perancangan mesin-mesin industri. Deepublish, 2018. 